\title{
CARACTERIZAÇÃO FÍSICA E QUÍMICA DOS FRUTOS DA UMBU-CAJAZEIRA (Spondias spp) EM CINCO ESTÁDIOS DE MATURAÇÃO, DA POLPA CONGELADA E NÉCTAR ${ }^{1}$
}

\author{
ELIZA DOROTEA POZZOBON DE ALBUQUERQUE LIMA', ${ }^{2,4}$ CARLOS ALBERTO DE \\ ALBUQUERQUE LIMA²; MAURO LUIZ ALDRIGUE'; PERLA JOANA SOUSA GONDIM ${ }^{3}$
}

\begin{abstract}
RESUMO - A umbu-cajazeira, no Brasil, apresenta boas potencialidades de cultivo e perspectivas de comercialização, o que objetivou este trabalho de avaliação da qualidade física e química dos frutos em cinco estádios de maturação classificados de acordo com o grau de cor da casca descritos como: fruto totalmente verde (1FTV), frutos com início de pigmentação (2FIP), frutos parcialmente amarelos (3FPA), frutos totalmente amarelos (4FTA), frutos totalmente amarelo-alaranjados (5FTAA), da polpa congelada e do néctar. As variáveis estudadas foram os atributos físicos: peso, diâmetro longitudinal, diâmetro transversal e rendimento em polpa, e químicos: vitamina $\mathrm{C}$, acidez total titulável sólidos solúveis totais, $\mathrm{pH}$ e a relação sólidos solúveis totais/acidez total titulável. Os frutos, no estádio de maturação comercial (4FTA), apresentaram os seguintes valores médio: rendimento de polpa de 55,75\%; pH de 2,08; SST de $11,25^{\circ}$ Brix; ATT de 1,77 g de ácido citrico/100g de polpa; SST/ATT de 6,39 e teor de vitamina C total de $17,75 \mathrm{mg} / 100 \mathrm{~g}$. A polpa congelada e o néctar mantiveram-se em condições estáveis em relação ao pH, SST, ATT e SST/ATT, durante 60 dias de armazenamento. Quanto ao teor de vitamina $\mathrm{C}$ total, a polpa congelada apresentou um decréscimo significativo, o que não ocorreu com o néctar.
\end{abstract}

Termos de indexação: Spondia spp, umbu-cajá, caracterização, polpa, néctar

\section{PHYSICAL AND CHEMICAL CHARACTERIZATION ON THE FRUITS OF UMBU-CAJAZEIRA (Spondias spp) IN FIVE RIPENING STAGES, FROZEN PULP AND NECTAR}

\begin{abstract}
In Brazil some tropical fruits present great potential for cultivation and perspective of commercialization, one of them is the "umbu-cajazeira". A research has been developed aiming to measure the physical and chemical quality of the frozen pulp and the nectar obtained from "umbu-cajazeira" fruits, which were classified in distinct maturation stages. The fruits were divided according to shell colour in five groups: fruit completely green (1FCG), fruit starting to change shell's colour (2FSCC), fruit partially yellow (3FPY), fruit totally yellow (4FTY), fruit totaly orange-like yellow. The variables analysed comprised the physical attributes - weight, longitudinal diameter, transversal diameter and pulp yield and the chemical parameters - vitamin C and total acidity (TA) expressed as citric acid, total soluble solids (TSS), $\mathrm{pH}$ and the rate total soluble solids/total acidity. The fruits in the commercial maturation stage (4FTY) presented the following average values: $55,7 \%$ pulp yield; $2,08 \mathrm{pH} ; 11,25$ total soluble solids; 1,77 total acidity; 6,39 TSS/TA and 17,75 $\mathrm{mg} / 100 \mathrm{~g}$ vitamin C. The frozen pulp and the nectar presented stable conditions when related to the $\mathrm{pH}$., TSS, TA and TSS/TA stored over a period of 60 days. However, the viatamin $\mathrm{C}$ content in the frozen pulp showed a significant decrease when stored under the same condition, fact that was not observed for the nectar.
\end{abstract}

Index terms: Spondia spp, umbu-cajá, characterization, frozen pulp, nectar.

\section{INTRODUÇÃO}

A umbu-cajazeira (Spondia spp) pertence à família Anacardiaceae e ao gênero Spondia considerado um híbrido natural entre o umbuzeiro e a cajazeira (Giacometti, 1993) e tem origem desconhecida, apresentando características de planta xerófita encontrada em plantios desorganizados disseminado em Estados do Nordeste. Na Paraíba, esta espécie se encontra distribuída do litoral ao sertão, sendo que, na região do Brejo, são encontrados os exemplares mais exuberantes (Lopes, 1997).
O fruto é caracterizado como uma drupa arredondada, de cor amarela, casca fina e lisa, com endocarpo chamado "caroço", grande, branco, suberoso e enrugado, localizado na parte central do fruto, no interior do qual se encontram os lóculos, que podem ou não conter uma semente. A umbu-cajazeira apresenta cerca de 90\% dos endocarpos desprovidos de sementes (Souza et al.,1997) o que torna inviável a sua propagação sexual, sendo tradicionalmente propagada pelo método vegetativo assexuado , através de estacas de $35 \mathrm{~cm}$ de comprimento e $1,5 \mathrm{~cm}$ de diâmetro (Lopes, 1997; Souza, 1998).

1 (Trabalho 106/2001). Recebido: 25/05/2001. Aceito para publicação: 13/02/2002. Trabalho realizado no Setor de Tecnologia de Alimentos-DSERCCA-UFPB.

2 Professores do DSER-CCA-UFPB - E-mail: epozzob @zaz.com.br - Rua: Eutiquiano Barreto 444 -Bairro Manaíra-João Pessoa-PB - CEP: 58038-

310 - Fone: 0xx83 - 247-1730

3 Professor do DTQA-CT-UFPB

4 Eng $^{\mathrm{a}}$ Agr ${ }^{\mathrm{a}}$ e aluna especial do Curso de Mestrado em Ciência de Alimentos-CT-UFPB.

Rev. Bras. Frutic., Jaboticabal - SP, v. 24, n. 2, p. 338-343, agosto 2002 
Os frutos possuem excelente sabor e aroma, boa aparência e qualidade nutritiva, muito consumidos na forma "in natura", apresentando rendimento médio de 55 a $65 \%$ em polpa, com potencial para a sua utilização na forma processada como polpa congelada, sucos, néctares e sorvetes.

A época de colheita, na Paraíba, ocorre no período de abril a julho. O método de colheita pode ser o manual, pois a umbu-cajazeira apresenta altura menor que a cajazeira, facilitando a coleta dos frutos, que devem ser colhidos nos estádios "verdosos" ou "de vez", tendo em vista serem classificados como climatéricos, o que proporciona uma melhor seleção dos frutos e qualidade de seus produtos. Os frutos maduros desprendem-se da planta e caem, ocasionando danos ao se chocarem com galhos e solo, podendo perder líquido e entrar em processo de fermentação, além de ficarem expostos ao ataque de insetos, deteriorando-se rapidamente.

Objetivando maior espaço na sua comercialização e apresentação no mercado consumidor, foram analisadas as característica físicas e físico-químicas em diferentes estádios de maturação em frutos de umbu-cajá, em amostras de polpa congelada obtida de frutos considerados no estádio de maturação comercial, bem como no produto de polpa processada para obtenção de néctar.

\section{MATERIAL E MÉTODOS}

O presente experimento foi conduzido no Laboratório de Tecnologia de Alimentos e Pós-Colheita do Centro Ciências Agrárias da Universidade Federal da Paraíba.

Foram utilizados umbu-cajás produzidos na região do Brejo Paraibano, no município de Areia-PB, no período de maio a junho de 2000. Os frutos foram colhidos manualmente em cinco estádios de maturação, levando em consideração a coloração externa, acondicionados de maneira a evitar escoriações e levados ao laboratório.

Após a seleção para eliminar os frutos danificados, foram uniformizados quanto ao tamanho e classificados quanto ao grau de amadurecimento, submetidos às análises e ao respectivo processamento. Os frutos foram classificados em cinco estádios de maturação, de acordo com o grau de cor da casca, utilizandose do procedimento através de seleção visual, separando-se os frutos em bandejas, utilizando-se da descrição demonstrada na Tabela 1 e Figura 1. Utilizaram-se frutos no estádio 4FTA, que demonstraram a potencialidade das características de maturação organoléptica completa na produção de polpa congelada e néctar.

De cada estádio, tomaram-se ao acaso 10 frutos para a caracterização física e química. As características físicas em relação aos frutos foram: peso, através de balança semi-analítica, diâmetros longitudinal (altura) e transversal (largura), obtidos por paquímetro, e rendimento de extração de polpa, subtraindose o peso da casca e semente do fruto inteiro.

As características determinadas nos frutos, polpa congelada e néctar foram: o $\mathrm{pH}$ através de $\mathrm{pHmetro}$ Digital DIGIMED; o teor de acidez total titulável (ATT) determinada segundo as normas analíticas do Instituto Adolfo Lutz (1985) e os resultados foram expressos em gramas de ácido cítrico/100g de polpa; sólidos solúveis totais (SST) determinados por refratometria, com os resultados expressos em ${ }^{\circ}$ Brix; a relação SST/ATT e o teor de vitamina $\mathrm{C}$ total (ácido ascórbico-AA + ácido desidroascórbicoDHAA) através do método titulométrico com 2,6dicloroindofenol (DFI) de acordo com Cunniff(1997), modificado com uso do ácido oxálico 5\%, como solução extratora. Para a determinação de vitamina $\mathrm{C}$ total, foi utilizada uma alíquota de 5 $\mathrm{ml}$ do filtrado e desenvolvendo a reação de redução do DHAA com DL-homocisteína, nas condições desenvolvidas por Aldrigue (1998). As análises com os frutos foram iniciadas aproximadamente 12 horas após a colheita, utilizando-se do suco obtido em liquidificador de uso doméstico.

TABELA 1- Classificação dos estádios de maturação do umbucajá de acordo com o grau da cor da casca

\begin{tabular}{ll}
\hline & \multicolumn{1}{c}{ Características } \\
\hline 1FTV & Fruto Totalmente Verde \\
2FIP & Fruto com Início de Pigmentação \\
3FPA & Fruto com Predominância Amarelo \\
4FTA & Fruto Totalmente Amarelo \\
5FTAA & Fruto Totalmente Amarelo-Alaranjado \\
\hline
\end{tabular}

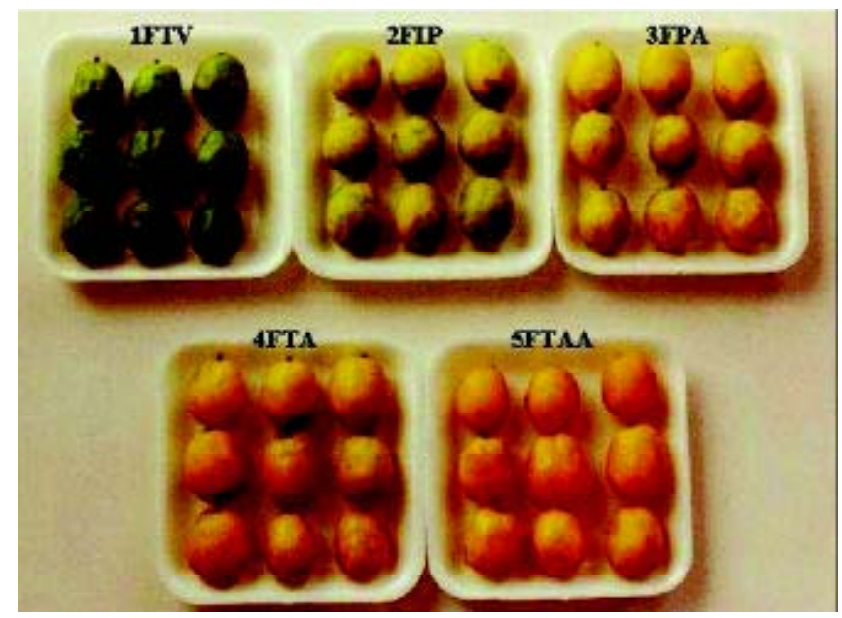

FIGURA 1- Grau de coloração dos umbu-cajás analisados.

\section{Polpa congelada}

O produto foi preparado com frutas inteiras, sadias, limpas, no estádio de maturação comercial (4FTA), com sabor e aroma agradáveis. A Figura 2 mostra etapas do processamento para a obtenção da polpa congelada. 


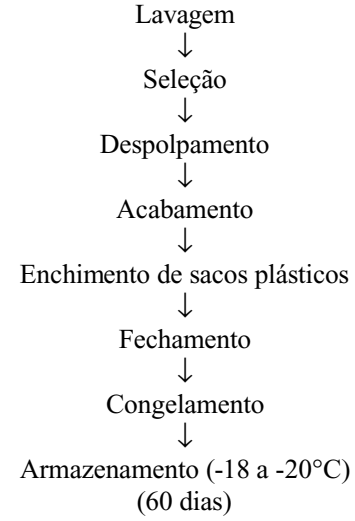

FIGURA 2- Fluxograma do processamento da polpa de umbucajá para produção de polpa congelada.

\section{Néctar}

Após a obtenção da polpa no estádio de maturação comercial (4FTA), foi formulado o néctar, com o seguinte fluxograma:

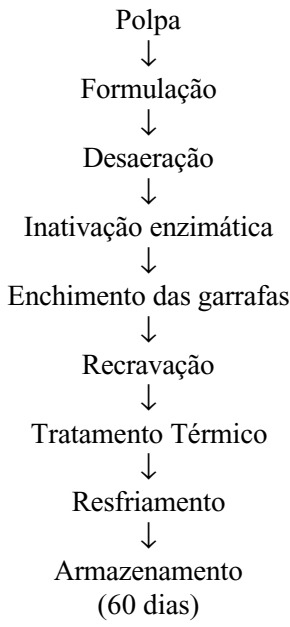

FIGURA 3 - Fluxograma do processamennto do néctar de umbucajá

O delineamento experimental foi o inteiramente casualizado, com 5 tratamentos e 3 repetições, com 3 frutos cada. As médias das variáveis foram submetidas à análise de variância e comparadas ao teste de Tukey, com probabilidade de erro de $5 \%$.

\section{RESULTADOS E DISCUSSÃO}

Os frutos da umbu-cajazeira apresentam boas características para industrialização na forma de polpa congelada, sucos, geléias, néctares e sorvetes, devido ao percentual médio de rendimento em polpa em frutos maduros (4FTA) de $55,75 \%$, conforme resultados encontrados na Tabela 2.

As mudanças de coloração de verde para amareloalaranjada evidenciadas nos diferentes estádios de maturação, apresentadas na Figura 1, estão associadas à diminuição dos pigmentos cloroplastídicos devido à degradação da clorofila da casca dos frutos de umbu-cajá no decorrer da sua maturação.

$\mathrm{O}$ pH manteve-se estável nos diferentes estádios de maturação dos frutos com os valores médios de 2,01 a 2,09 (Tabela 3), podendo-se observar que as polpas de umbu-cajás no estádio maduro (4FTA) apresentaram valores de $\mathrm{pH}$ um pouco abaixo dos encontrados em outros frutos do gênero Spondias como cajá apresentando $\mathrm{pH}$ de 3,30 (Aldrigue, 1988), 2,75 (Lima et al., 1990) 2,4 a 3,0 ( Sacramento \& Souza, 2000), umbu-azedo com 2,26 e umbu-doce com 2,28 (Costa, 1999). Os valores médios de $\mathrm{pH}$ e acidez total titulável encontrados favorecem a sua conservação, não havendo necessidade da adição de ácido cítrico na formulação, no processamento de néctar, para evitar o crescimento de leveduras.

Os conteúdos de SST apresentados na Tabela 3 aumentaram em função dos estádios de maturação, havendo diferença significativa apenas para frutos $1 \mathrm{FTV}$ em relação aos demais graus de maturação, constatando-se valores médios de 11,25 ( ${ }^{\circ}$ Brix), sendo estatisticamente semelhantes. Os índices alcançados estão próximos dos limites a que chegaram Noronha et al. (2000), de 11,04 e 12,88\%. Os teores de ATT expressos em gramas de ácido cítrico mostraram-se mais elevados nos frutos 1FTV e decrescentes com o aumento da maturação, sofrendo variações significativas em função do estádio de maturação, obtendo-se os valores de 2,40 a $1,55 \mathrm{~g}$ de ácido cítrico/100g, com comportamento e resultados semelhantes aos apresentados por Costa (1998) com cajás . A razão SST/ATT aumentou consideravelmente entre os estádios dos frutos 2FIP e frutos 3FPA, na medida em que os frutos apresentaram maior grau de amadurecimento (Tabela 3). A tendência da redução da ATT e o aumento dos SSTs e da relação SST/ATT, após completa expansão do fruto, evidenciaram que o umbu-cajá pode ser colhido a partir dos frutos 3FPA para armazenamento ou consumo quase imediato.

Os valores de vitamina $C$ total apresentados na Tabela 4 demonstram que os frutos atingiram o teor máximo no estádio de maturação do fruto parcialmente amarelo (3FPA) com $18,35 \mathrm{mg} /$ $100 \mathrm{~g}$, apresentando perda mais intensa nos frutos totalmente

TABELA 2 - Características físicas de umbu-cajá em cinco estádios de maturação

\begin{tabular}{lcccc}
\hline \multicolumn{1}{c}{ Amostras } & $\begin{array}{c}\text { Diâmetro } \\
\text { Longitudinal }(\mathrm{cm})\end{array}$ & $\begin{array}{c}\text { Diâmetro Transversal } \\
(\mathrm{cm})\end{array}$ & $\begin{array}{c}\text { Peso } \\
(\mathrm{g})\end{array}$ & $\begin{array}{c}\text { Rendimento de } \\
\text { Polpa (\%) }\end{array}$ \\
1FTV & $3,75 \mathrm{a}$ & $3,40 \mathrm{a}$ & $17,77 \mathrm{c}$ & $25,04 \mathrm{~d}$ \\
2FIP & $3,85 \mathrm{a}$ & $2,65 \mathrm{~b}$ & $19,60 \mathrm{a}$ & $49,38 \mathrm{c}$ \\
3FPA & $3,60 \mathrm{a}$ & $2,45 \mathrm{~b}$ & $19,07 \mathrm{~b}$ & $52,97 \mathrm{~b}$ \\
4FTA & $3,65 \mathrm{a}$ & $2,90 \mathrm{~b}$ & $22,30 \mathrm{a}$ & $55,75 \mathrm{a}$ \\
5FTAA & $3,50 \mathrm{a}$ & $2,60 \mathrm{~b}$ & $19,10 \mathrm{~b}$ & $52,60 \mathrm{~b}$ \\
\hline DMS & 0,70 & 064 & 1,01 & 1,27 \\
CV\% & 4,80 & 5,76 & 1,29 & 0,67 \\
\hline
\end{tabular}

Médias seguidas de mesma letra não diferem significativamente entre si, ao nível de $5 \%$ de probabilidade, pelo Teste de Tukey. 
TABELA 3 - Características químicas do fruto umbu-cajá em cinco estádios de maturação

\begin{tabular}{lcccc}
\hline \multicolumn{1}{c}{ Amostras } & $\mathrm{pH}$ & $\begin{array}{c}\text { SST } \\
\left({ }^{\circ} \mathrm{Brix}\right)\end{array}$ & $\begin{array}{c}\text { ATT } \\
(\%)\end{array}$ & $\begin{array}{c}\text { SST/ATT } \\
(\%)\end{array}$ \\
\hline 1FTV & $2,01 \mathrm{a}$ & $9,10 \mathrm{~b}$ & $2,40 \mathrm{a}$ & $3,80 \mathrm{e}$ \\
2FIP & $2,02 \mathrm{a}$ & $10,65 \mathrm{a}$ & $2,20 \mathrm{~b}$ & $4,85 \mathrm{~d}$ \\
3FPA & $2,09 \mathrm{a}$ & $10,70 \mathrm{a}$ & $1,63 \mathrm{~d}$ & $6,57 \mathrm{~b}$ \\
4FTA & $2,00 \mathrm{a}$ & $11,25 \mathrm{a}$ & $1,77 \mathrm{c}$ & $6,39 \mathrm{c}$ \\
5FTAA & $2,06 \mathrm{a}$ & $11,25 \mathrm{a}$ & $1,55 \mathrm{e}$ & $7,51 \mathrm{a}$ \\
\hline DMS & 0,12 & 0,05 & 0,25 & 0,47 \\
CV\% & 1,53 & 0,67 & 3,36 & 0,20 \\
\hline
\end{tabular}

Médias seguidas de mesma letra não diferem significativamente entre si, ao nível de $5 \%$ de probabilidade, pelo Teste de Tukey.

TABELA 4 - Teor de vitamina $C$ total em frutos de umbu-cajás em diferentes estádios de maturação

\begin{tabular}{lccc}
\hline \multicolumn{1}{c}{} & Vitamina C (mg/100g) \\
\hline \multicolumn{1}{c}{ A mostras } & A A & D H A A & Total \\
\hline 1 F T V & $13,70 \mathrm{a}$ & $0,55 \mathrm{~b}$ & $14,25 \mathrm{a}$ \\
F P & $12,35 \mathrm{a}$ & $4,10 \mathrm{a}$ & $16,45 \mathrm{a}$ \\
3 F A & $13,20 \mathrm{a}$ & $5,15 \mathrm{a}$ & $18,35 \mathrm{a}$ \\
4 F A & $13,35 \mathrm{a}$ & $4,40 \mathrm{a}$ & $17,75 \mathrm{a}$ \\
5 F A A & $8,60 \mathrm{~b}$ & $4,10 \mathrm{a}$ & $12,90 \mathrm{a}$ \\
\hline D M S & 2,51 & 3,29 & 5,52 \\
C V & 5,13 & 22,43 & 8,65 \\
\hline
\end{tabular}

Médias seguidas de mesma letra não diferem significativamente entre si, ao nível de $5 \%$ de probabilidade, pelo Teste de Tukey.

TABELA 5- Características químicas das polpas congeladas de umbu-cajá no estádio de maturação do fruto totalmente amarelo (4FTA)

\begin{tabular}{lcccc}
\hline Tempo/dias & pH & $\begin{array}{c}\text { S S T } \\
\left({ }^{\circ} \mathrm{B} \mathrm{rix}\right)\end{array}$ & $\begin{array}{c}\text { A T T } \\
(\%)\end{array}$ & $\begin{array}{c}\text { S T /A T T } \\
(\%)\end{array}$ \\
\hline 0 & $2,13 \mathrm{ab}$ & $11,25 \mathrm{a}$ & $1,78 \mathrm{a}$ & $6,29 \mathrm{a}$ \\
30 & $2,17 \mathrm{a}$ & $11,00 \mathrm{~b}$ & $1,75 \mathrm{a}$ & $6,28 \mathrm{a}$ \\
60 & $2,11 \mathrm{~b}$ & $11,00 \mathrm{~b}$ & $1,75 \mathrm{a}$ & $6,28 \mathrm{a}$ \\
\hline D M S & 0,05 & 0,17 & 0,07 & 0,18 \\
C V \% & 0,66 & 0,37 & 0,96 & 0,72 \\
\hline
\end{tabular}

Médias seguidas de mesma letra não diferem significativamente entre si, ao nível de $5 \%$ de probabilidade, pelo Teste de Tukey.

TABELA 6 - Características químicas do néctar de umbu-cajá no estádio de maturação do fruto totalmente amarelo (4FTA)

\begin{tabular}{lcccc}
\hline \multicolumn{1}{c}{ Tempo/dias } & p H & $\begin{array}{c}\text { S S T } \\
\left({ }^{\circ} \text { Brix }\right)\end{array}$ & $\begin{array}{c}\text { A T T } \\
(\%)\end{array}$ & $\begin{array}{c}\text { S T /A T T } \\
(\%)\end{array}$ \\
\hline 0 & $2,29 \mathrm{~b}$ & $18,30 \mathrm{~b}$ & $0,45 \mathrm{~b}$ & $40,66 \mathrm{a}$ \\
30 & $2,39 \mathrm{a}$ & $19,00 \mathrm{a}$ & $0,50 \mathrm{a}$ & $38,05 \mathrm{~b}$ \\
60 & $2,41 \mathrm{a}$ & $19,00 \mathrm{a}$ & $0,49 \mathrm{a}$ & $38,38 \mathrm{~b}$ \\
\hline D M S & 0,04 & 0,34 & 0,02 & 1,51 \\
C V \% & 0,42 & 0,44 & 1,19 & 0,93 \\
\hline
\end{tabular}

Médias seguidas de mesma letra não diferem significativamente entre si, ao nível de $5 \%$ de probabilidade, pelo Teste de Tukey.

amarelo-alaranjados (5FTAA) com início da senescência, com comportamento semelhante aos observados na maioria dos frutos tropicais estudados. $\mathrm{O}$ valor de vitamina $\mathrm{C}$ total obtido nos frutos, no estádio 4FTA de $17,75 \mathrm{mg} / 100 \mathrm{~g}$, está compatível com o resultado encontrado por Aldrigue (2000) com frutos obtidos no comércio. A diferença significativa que influiu para que o CV alcançasse $22,43 \%$ na determinação do DHAA, no estádio 1FTV, demonstra que, neste estádio, a forma predominante é o AA que, durante o processo de amadurecimento, se oxida, mantendo-se em equilíbrio com o DHAA.

Os teores de SST para polpas comercializadas congeladas de cajá (fruta com características bastante semelhantes) apresentam média de 7,67\% com valores máximos e mínimos de $14,00 \%$ a 2,00\%, respectivamente, como apresentam Bastos et al.(1999), não atendendo ao padrão para suco de cajá, conforme a legisla- ção atual, que estabelece valor mínimo para teor de sólidos solúveis totais de $8,00 \%$. Portanto, os teores médios de $11,00 \%$ de SST, apresentados na Tabela 5, demonstram que a polpa de umbucajá congelada, embora sem legislação específica, possui valores superiores a $8,00 \%$, podendo ser utilizada para produção de suco.

A Tabela 6 apresenta os resultados das análises químicas do néctar do umbu-cajá armazenado durante 60 dias, podendo ser observada ligeira mudança das características no primeiro mês de armazenamento, permanecendo estável no restante do período, não apresentando mudanças quanto ao $\mathrm{pH}, \mathrm{SST}$, ATT e SST/ATT.

$\mathrm{O}$ teor de vitamina $\mathrm{C}$ total do néctar é significativamente menor devido à diluição da polpa na sua formulação, ação da temperatura na inativação enzimática e na operação física de 
TABELA 7- Teor de vitamina $C$ total de polpa congelada e néctar de umbu-cajá processado no estádio de maturação do fruto totalmente amarelo (4FTA).

\begin{tabular}{|c|c|c|c|}
\hline \multicolumn{4}{|c|}{ Vitam ina $\mathrm{C}(\mathrm{mg} / 100 \mathrm{~g})$} \\
\hline A mostras & $\mathrm{A} \mathrm{A}$ & D H A A & Total \\
\hline Polpa congelada & $8,35 \mathrm{a}$ & $3,25^{\mathrm{a}}$ & $11,60 \mathrm{a}$ \\
\hline Néctar & $2,25 \mathrm{~b}$ & $2,35 \mathrm{~b}$ & $4,60 \mathrm{~b}$ \\
\hline $\mathrm{D} \mathrm{M} \mathrm{S}$ & 0,30 & 0,30 & 0,60 \\
\hline $\mathrm{C} \mathrm{V} \%$ & 1,33 & 2,53 & 1,75 \\
\hline
\end{tabular}

Médias seguidas de mesma letra não diferem significativamente entre si, ao nível de $5 \%$ de probabilidade, pelo Teste de Tukey.

TABELA 8 - Teor de vitamina $\mathrm{C}$ da polpa congelada durante o armazenamento

\begin{tabular}{|c|c|c|c|}
\hline \multirow[t]{2}{*}{ Tempo/dias } & \multicolumn{3}{|c|}{ Vitam ina $\mathrm{C}(\mathrm{mg} / 100 \mathrm{~g})$} \\
\hline & $\mathrm{A} \mathrm{A}$ & D H A A & Total \\
\hline 0 & $8,35 \mathrm{a}$ & $3,25 \mathrm{a}$ & $11,60 \mathrm{a}$ \\
\hline 60 & $5,15 b$ & $2,85 \mathrm{~b}$ & $8,00 \mathrm{~b}$ \\
\hline $\mathrm{D} \mathrm{M} \mathrm{S}$ & 0,30 & 0,30 & 0,43 \\
\hline $\mathrm{C} \mathrm{V} \%$ & 1,05 & 2,32 & 1,02 \\
\hline
\end{tabular}

Médias seguidas de mesma letra não diferem significativamente entre si, ao nível de $5 \%$ de probabilidade, pelo Teste de Tukey.

TABELA 9 - Teor de vitamina $\mathrm{C}$ do néctar durante o armazenamento

\begin{tabular}{lccc}
\hline Tempo/dias & \multicolumn{2}{c}{ Vitamina C (mg/100g) } \\
\hline & A A & D H A A & Total \\
\hline 0 & $2,25 \mathrm{a}$ & $2,35 \mathrm{a}$ & $4,60 \mathrm{a}$ \\
60 & $2,45 \mathrm{a}$ & $2,40 \mathrm{a}$ & $4,85 \mathrm{a}$ \\
& & & 0,48 \\
D M S & 0,30 & 0,21 & 2,37 \\
\hline
\end{tabular}

Médias seguidas de mesma letra não diferem significativamente entre si, ao nível de $5 \%$ de probabilidade, pelo Teste de Tukey.

desaeração (Tabela 7).

O tempo de armazenamento de 60 dias da polpa congelada do umbu-cajá apresentou uma diminuição de vitamina $\mathrm{C}$ total em $31 \%$, o que demonstra ser totalmente viável o seu aproveitamento como produto comercial (Tabela 8).

Quanto ao néctar, o teor de vitamina C total (Tabela 9) durante o período de 60 dias de estocagem à temperatura ambiente $\left(28 \pm 3^{\circ} \mathrm{C}\right)$ manteve-se estável, e a diferença para um maior valor está dentro da faixa de variação determinado.

\section{CONCLUSÕES}

1. O aumento da relação SST/ATT, decorrente da redução de ATT, e o aumento dos SSTs apresentados nos estádios de maturação dos frutos parcialmente amarelos (3FPA) e frutos totalmente amarelos (4FTA) evidenciam as condições de colheita, armazenamento e consumo imediato.

2. A determinação física do umbu-cajá dos estádios 3FPA e 4FTA demonstrou condições adequadas para comercialização por apresentar rendimento em polpa acima de $50 \%$.

3. O teor de ácido desidroascórbico (DHAA) variou de 4,0 a $32,0 \%$ do total de vitamina $\mathrm{C}$ do umbu-cajá nos 5 estádios de maturação, apresentando teor de vitamina C total (AA+DHAA) razoável, sendo que a polpa congelada do umbu-cajá apresen- tou um decréscimo de $31 \%$ do teor de vitamina $\mathrm{C}$ total ao final de 60 dias de armazenamento, não ocorrendo, porém, nenhuma perda com o néctar.

4. O umbu-cajá caracteriza-se como uma matéria-prima que apresenta um grande potencial para seu aproveitamento comercial, apresentando-se como naturalmente ácida, com $\mathrm{pH}$ ao redor de 2,08 , de forma a tornar o meio impróprio ao desenvolvimento de microrganismos.

\section{REFERÊNCIAS BIBLIOGRÁFICAS}

ALDRIGUE, M.L. Desenvolvimento e validação de metologia analítica, utilizando a CLAE, para determinação de vitamina C em frutas e seus principais produtos. 1998,160 f. Tese (Doutorado em Ciência de Alimentos) - Faculdade de Engenharia de Alimentos, Universidade Estadual de Campinas, Campinas, 1998.

ALDRIGUE, M,L.; TORRES, T.S.C.; FERREIRA, F.T. Teor de vitamina $C$ e características físicas do umbu-cajá. In: CONGRESSO BRASILEIRO DE CIÊNCIA E TECNOLOGIA DE ALIMENTOS. 17., 2000. Fortaleza. Livro de resumos. v.2, n.5, p.123.

BASTOS, M.S.R.; FEITOSA, T.; OLIVEIRA, M.E.B. Análise qualitativa e tecnológica da agroindústria de polpa de fruta na re- 
gião Nordeste. Revista Brasileira de Fruticultura. Jaboticabal, v.21,n.3,p.359-364, 1999.

COSTA, N.P. Caracterização de frutos e germinação de endocarpos de umbu (Spondias tuberosa Arruda Câmara). 1999. 64 f. Dissertação (Mestrado no Curso de Produção Vegetal) Universidade Federal da Paraíba, Areia, 1999.

COSTA, N.P. Desenvolvimento, maturação e conservação póscolheita de frutos da cajazeira (Spondias mombin L.). 1998, 97 f. Dissertação (Mestrado no Curso em Produção Vegetal) - Universidade Federal da Paraíba, Areia, 1998.

CUNNIF, P. Official Methods of Analiysis of AOAC International. 16.ed. Gaithersburg: AOAC-International, 1997. v. 2.

GIACOMETTI, D.C. Recursos genéticos de fruteiras nativas do Brasil. In: SIMPÓSIO NACIONAL DE RECURSOS GENÉTICOS DE FRUTEIRAS NATIVAS, 1992, Cruz das Almas, BA. Anais... Cruz das Almas: Embrapa-CNPMF, 1993. p.13-27.

INSTITUTO ADOLFO LUTZ. Métodos físicos e químicos para análise de alimentos. São Paulo-SP: 1985. 533p.

LIMA, E.D.P.A.; CARDOSO,E.de A.; SILVA,H.,LIMA,C.A.A.;
SILVA,A.Q.da. Características tecnológicas de frutos do gênero Spondias, família Anacardiaceae. In: REUNIÃO NORDESTINA DE BOTÂNICA, 1.,1990, Recife. Anais.. p.109.

LOPES, W.F. Propagação assexuada de cajá (Spondias mombin L.) e cajá-umbu (Spondias spp) através de estacas. Areia, 1997. 47p. (Relatório final PIBIC - CNPq).

NORONHA, M.A.S.; NORONHA, M.A.S.; CARDOSO, E.A.; MENEZES, J.B.; GÓIS, V.A. Características físico-químicas de umbu-cajá (Spondias spp) provenientes dos pólos Baixo Jaguaribe (CE) e Assu-Mossoró(RN). In: CONGRESSO BRASILEIRO DE FRUTICULTURA. 16., 2000. Fortaleza. Resumos... Sociedade Brasileira de Fruticultura, 2000.

SACRAMENTO, C.K.; SOUZA, F.X. Cajá (Spondias mombin L.) Jaboticabal: Funep, 2000. 42p. (Série Frutas Nativas, 4).

SOUZA, F.X. Spondias agroindustriais e os seus métodos de propagação. Fortaleza: EMBRAPA-CNPAT/SEBRAE/CE, 1998. 28p. (Documento, 27).

SOUZA, F.X. de.; SOUZA, F.H.L.; FREITAS, J.B.S. Caracterização morfológica de endocarpos de umbu-cajá. In: CONGRESSO NACIONAL DE BOTÂNICA, 48., 1997, Crato, CE. Resumos... Fortaleza: SBB/BNB, 1997.p.121. 